\title{
A Heuristics Framework for Semantic Subscription Processing
}

\author{
Martin Murth and eva Kühn \\ Vienna University of Technology \\ Institute of Computer Languages, \\ Space Based Computing Group \\ Argentinierstraße 8, 1040 Vienna, Austria \\ \{mm, eva @complang.tuwien.ac.at
}

\begin{abstract}
The increasing adoption of semantic web technology in application scenarios with frequently changing data has imposed new requirements on the underlying tools. Reasoning algorithms need to be optimized for the processing of dynamic knowledge bases and semantic frameworks have to provide novel mechanisms for detecting changes of knowledge. Today, the latter is mostly realized by implementing simple polling mechanisms. However, this implies client-side post-processing of the received results, causes high response times and limits the overall throughput of the system. In this paper, we present a heuristics framework for realizing a subscription mechanism for dynamic knowledge bases. By analyzing similarities between published information and resulting notifications, heuristics can be employed to "guess" subsequent notifications. As testing the correctness of guessed notifications can be implemented efficiently, notifications can be delivered to the subscribers in an earlier processing phase and the system throughput can be increased. We experimentally evaluate our approach based on a concrete application scenario.
\end{abstract}

Keywords: Semantic subscription processing, heuristics framework, continuous queries, similarity heuristics, incremental result set updates.

\section{Introduction}

The employment of semantic web technology in an ever increasing number of different application areas has imposed new requirements on the underlying tools and frameworks. Besides the traditionally high requirements on storing, querying and reasoning about large static data sets, efficient processing of more dynamic data has become a central issue in many application scenarios, e.g., knowledge distribution for synchronization or clustering [1], semantically-enabled data integration [2], knowledge monitoring [3], semantic message routing [4-5], and knowledge-driven coordination [6-7].

The new requirements can generally be divided into two groups. First, reasoning algorithms and engines need to be optimized for the processing of frequently changing data. Different approaches have already been investigated in this field of 
research. In [8], for instance, an incremental reasoning technique for dynamic ABoxes is described, which allows for improvements of reasoning about $\mathcal{S H O} \mathcal{Q}$ and $\mathcal{S H} \mathcal{H} \mathcal{Q}$ description logics [9] of "up to three orders of magnitude".

Second, semantic frameworks need to provide novel mechanisms for detecting changes of knowledge in a knowledge base. Today, this is often realized by implementing a simple client-side polling mechanism. The client system executes the query, retrieves the full result set and compares it with previously received result sets. Although the client is actually only interested in relevant updates, it needs to store the entire query result sets and analyze potentially large amounts of data.

Accounting for this, we developed a lightweight framework that implements a simple subscription mechanism for semantic repositories. Whenever data is added to the repository, subscribers are notified about new results of the registered queries. With this approach, the client does not need to store and post-process the received results and the amount of data that needs to be transferred between the semantic repository and the client is minimized. However, it is still necessary to execute all registered queries whenever new data is added. This often causes high response times and limits the overall throughput of the system (cf. [6]).

In this paper, we present a mechanism for reducing the time required to notify subscribers by applying heuristics on the published data for finding new query results. The core idea is based on the following observation: While adding new data to the knowledge base often entails a costly reasoning and query execution task to find new query results, the contents of the published graphs and the new query results can frequently be correlated following simple patterns. Hence, finding such patterns would allow the system to "guess" possible new results and to test whether they are valid in the new system state. Testing of possible results can be implemented efficiently and the valid results can be sent to the subscriber already before the usually much more expensive query is executed. Furthermore, if the heuristic is able to find the majority of new results, query execution can even be skipped for several processing cycles. This decreases the average time required to process a publication request and therefore increases the throughput of the semantic subscription system.

The rest of this paper is organized as follows: The remainder of Section 1 discusses related work in this field. Section 2 introduces SENS (Semantic Event Notification Service), our subscription processing framework for semantic repositories, and describes an extension for heuristic result set update algorithms. A simple heuristic for this framework is developed in Section 3. Section 4 evaluates the approach based on a concrete application scenario. Conclusions and future work are presented in Section 5 .

\subsection{Related Work}

The work presented in this paper was motivated by experiences with application development using semantic tuplespace technology. Semantic tuplespaces (e.g., sTuples [10], TSC [11], Semantic Web Spaces [12], TripCom [13]) are usually built upon a semantic repository and implement a Linda-based [14] or a publish/subscribebased interface to access the stored knowledge. Clients interact with each other by adding knowledge to the knowledge base and by retrieving notifications about events they have subscribed for. Although the proposed systems allow for elegant solutions 
of many complex coordination problems (e.g. [15]), the implementation of real world applications showed that the existing systems do not perform well for bigger knowledge bases as the continuous execution of queries causes a too high load on the server. In the TripCom project, mechanisms are being developed for clustering and distributing the data to multiple server instances. While this approach aims at improving scalability by intelligently distributing the load, our approach focuses on improving the performance of a single server instance. Thus, both approaches complement each other.

Many semantic repositories and frameworks implement a simple subscription mechanism. Jena [16] and Sesame [17], for example, implement listener interfaces that generate events whenever new triples are added to the repository. However, they do not support expressive subscription languages and notify listeners only about explicitly inserted triples. Consequently, subscribers are not notified about inferred information which is one of the crucial advantages of communicating via a shared knowledge base.

Some RDF query engines (e.g. [18-19]) employ caches for improving the performance of query answering. While this technique leads to good results for querying static data, it is hardly applicable for highly dynamic knowledge bases as the caches are invalidated each time new data is published.

Several algorithms for continuous queries on RDF data have been proposed in [4-5,20-21]. However, these algorithms require RDF triples as input data, which requires to instantiate all triples at least in memory. For applications that employ ontologies with extensive use of transitive properties or classification conditions, this might cause the instantiation of a huge, possibly exponentially growing number of RDF triples. In contrast, our approach builds upon repeated query executions but tries to find the optimal intervals and to reduce the number of execution cycles. Thus, triples only need to be instantiated if they are requested by the reasoning or the query engine. Moreover, the heuristics work independently of the employed query engine and reasoning technology.

\section{A Heuristics Framework for Semantic Subscriptions}

In this section we briefly introduce the subscription framework SENS and present a framework extension for heuristic result set update algorithms.

\subsection{SENS - Semantic Event Notification Service}

SENS (Semantic Event Notification Service) [6] is a framework for implementing and controlling complex and heterogeneous application scenarios by leveraging the reasoning capabilities of logic-based reasoning systems. Software clients can publish data at SENS and subscribe to changes of particular parts of the inferable knowledge. In contrast to conventional publish/subscribe systems, the published data is not directly forwarded to the subscribers but is, in a first step, added to the system's knowledge base. Afterwards, the system evaluates the new state of the knowledge base and notifies the subscribers about the according updates.

SENS is accessed via the API shown in Listing 1. 
Listing 1. SENS API (Java)

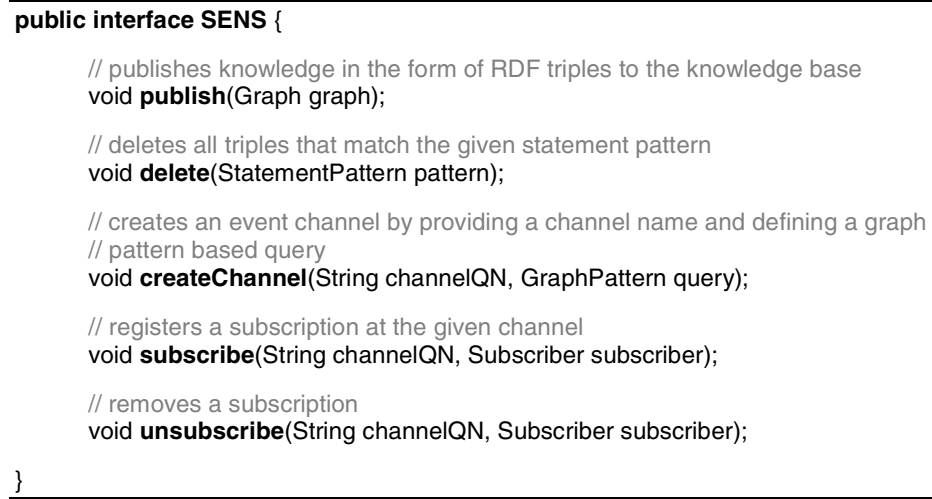

Clients can add knowledge to SENS in form of an RDF graph data structure (publish). The system tries to infer new knowledge right after the publication operation.

RDF triples can also be removed from the knowledge base by providing a statement pattern matching the triples to be deleted (delete). The deletion of triples also removes the knowledge that has been inferred from these triples. Previously inferred knowledge is thus not invalidated, but it is no longer guaranteed to be true.

For being notified about changes of knowledge, clients have to subscribe to a semantic event channel via its qualified name (subscribe/unsubscribe). A semantic event channel generates notifications about changes of a part of a knowledge base that has to be defined by means of a query statement (createChannel). As state-of-the-art semantic repositories implement a variety of different query languages, the current version of SENS only accepts query statements formulated as simple graph patterns as these can be expressed with most existing RDF query languages. However, it is planned to add support for SPARQL [22] queries in the next version. Whenever the knowledge base is updated, SENS executes the channels' queries and tags the returned bindings. If it finds bindings that were not contained in the previous result set of a particular channel (i.e., are not tagged yet), the subscribers are notified about these new bindings. Notifications are sent to the subscriber as variable bindings for the channel's query and can be accessed in tabular form or as variable substitutions of the query's graph pattern (analogous to SPARQL select and construct forms).

One of the main advantages of employing a semantic subscription framework like SENS is the high expressivity of the subscription mechanism. The use of logic-based reasoning allows detecting and reacting to complex dependencies between distributed clients which are not explicitly modeled in the application scenario but which can be inferred from the provided data. For describing the same dependencies using a query on a database or an event stream [23], one would require, e.g., nested subqueries, constraint evaluation, special treatment of null values, explicit case differentiations, and client-side post-processing of the received results. Besides this, the semantic publish/subscribe interaction pattern facilitates strong decoupling of the communicating clients with respect to time (clients do not need to be connected to the system at the same time), reference (clients do not need to know each other 
explicitly), and data schema (clients do not rely on homogenous data structures and semantics). This increases flexibility in application design and allows for more autonomous system architectures.

\subsection{An Extension for Heuristic Query Result Update Algorithms}

For the improvement of notification times and throughput of SENS, we developed a framework extension for heuristic query result set update algorithms. These algorithms try to find new query results by analyzing the history of published graphs and found notifications, but they do not execute the actual query itself.

As notification time $\delta$ notify $\left._{i . j}\right)$ of a notification ${ }^{1}$ notify $_{i . j}$, we define the time between the client-side invocation of the publish operation $p u b_{i}$ that triggered the notification and the client-side reception of the notification. Accordingly, the average notification time $\delta(C)$ for a channel $C$ is defined as the average of all notification times of those notifications that were delivered to the subscribers of a channel during their registration time.

Generally, the performance of the system is determined by the performance of all supported operations (publish, delete, createChannel, un/subscribe). However, for the applications we investigated, only the efficiency of publication processing had a significant impact on the overall system performance. Consequently, we define the throughput $\mu(S)$ of a semantic subscription system $S$ as the number of publication requests that can be processed (i.e., publication and notification of all concerned subscribers) within a certain time unit.

For the development of the framework extension, we had to adapt the processing model of SENS. The original processing phases executed after each publication operation are illustrated in Figure 1.

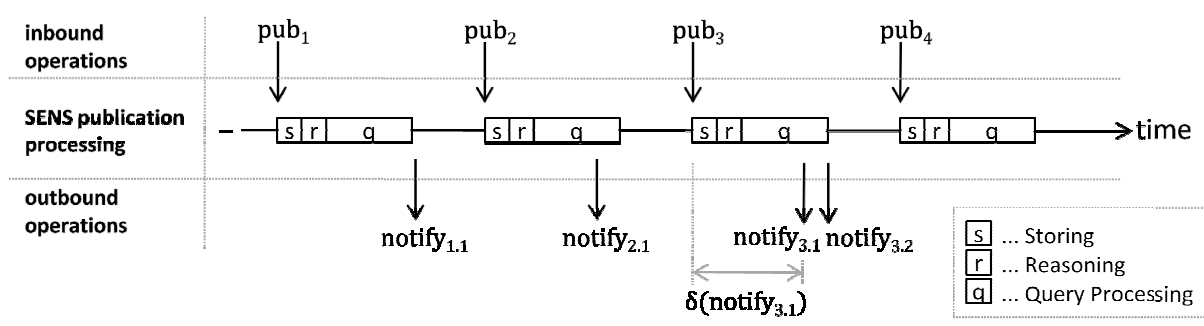

Fig. 1. SENS publication processing

When a publication request is issued to SENS (e.g., pub $b_{3}$ ), the contained graph is first stored (s) in the connected RDF repository. Then a reasoning engine is employed to infer new knowledge (r) and finally, SENS executes the queries (q) of the subscriptions of all defined channels. Depending on the query and the employed RDF repository, the execution of the query may involve additional reasoning on the knowledge base. Whenever a new binding is found, a notification is immediately sent to the concerned subscribers (e.g., notify $y_{3.1}$ ).

\footnotetext{
${ }^{1}$ Notify operations are given with two subscript numbers. The first one refers to the publication operation that triggered the notification and the second one is used to distinguish notifications that were triggered by the same publication operation.
} 
The main benefit of employing a heuristic algorithm for finding potential bindings of subscriptions is that testing single bindings takes considerably less time than the complete execution of a query. The system only has to verify whether the bound triples of the graph pattern also exist in the new state of the RDF repository.

Figure 2 illustrates the additional phases of the extended processing model.

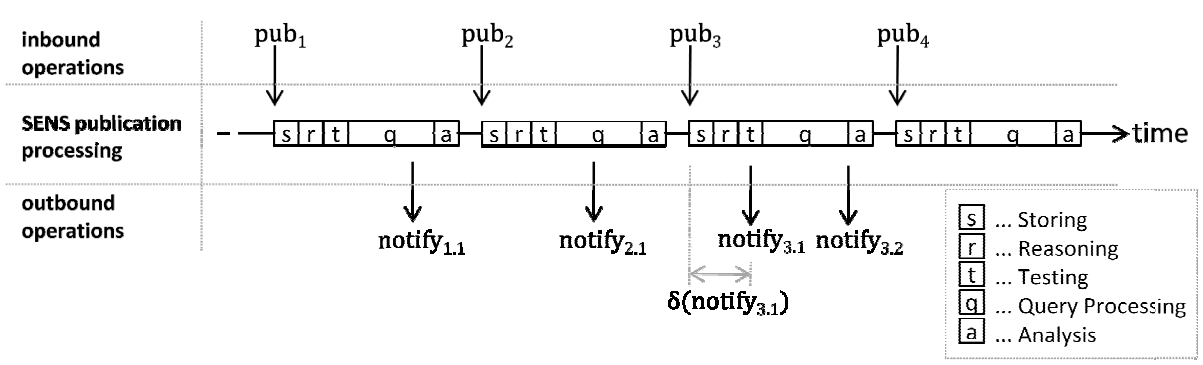

Fig. 2. SENS publication processing extension for result set update algorithms

Like in the original processing model, the content of a publication request is first stored (s) in the RDF repository and the reasoning engine tries to infer new knowledge (r). Afterwards, the heuristic analyzer component of SENS is provided with the new graph and returns a list of candidate bindings for each defined channel. SENS tests (t) for each candidate binding whether it represents a new notification event, i.e., whether it is a valid binding for the defined query and has not yet been delivered to the subscribers. If this is the case, subscribers are immediately notified about the new binding. In the next step, the standard query processing task $(\mathbf{q})$ is executed in order to reliably find all new bindings that have not been discovered in the testing phase. Finally, the heuristic analyzer component tries to adjust and improve its heuristic method for subsequent requests. It therefore searches for further correlations between published graphs and resulting notifications (a) based on all found notifications and the history of preceding publication operations.

\section{Simple Similarity Heuristic}

In the following, we present a result set update heuristic for the previously described framework. The algorithm should serve as a proof of concept rather than a final solution and should motivate the development of more advanced algorithms. We will illustrate the algorithm's functionality by means of a simplified example of a data integration application. In the application scenario, a semantic repository is used to manage organizational and reference data of a university, e.g., name, address, telephone number, courses, publications, etc. of students and employees. A number of (heterogeneous) systems are storing and retrieving data from the shared repository. As several different terminologies are being used, an ontology is employed that maps concepts of one terminology to semantically equivalent concepts of the others. By implementing client-to-client communication via publishing and subscribing to 
knowledge at SENS, data of different data schemas is translated automatically and transparently for the client.

In the example, a client registers the following subscription in order to be notified whenever new information about a Course of a Lecturer becomes available:

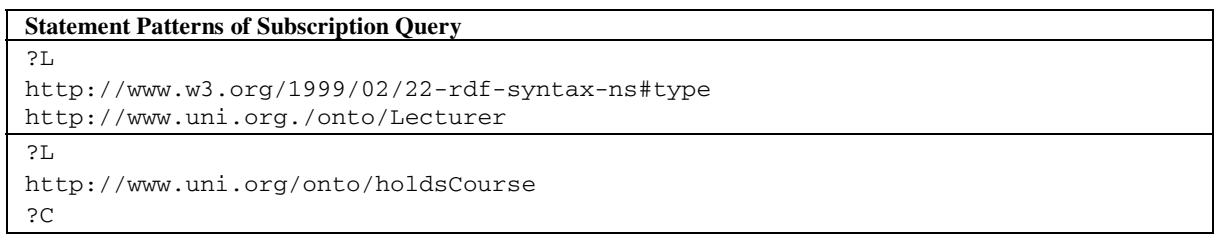

Each time a new binding for this query is found, the binding and the previously published graph are assigned to a particular binding and graph category. The following two tables show a published graph consisting of one single triple and a new binding resulting from the according publication operation. Note that in the ontology of the example, an individual is classified as Lecturer, if it teaches at least one course. The role teaches is defined to be the same as holdsCourse.

\begin{tabular}{|c|c|}
\hline Published Graph $^{2}$ & Number of Elements \\
\hline http://www.uni 1.org/dep 1/Professor 137 & 9 \\
\hline$\overline{\text { http }}: / / \overline{w w w} \cdot \overline{\text { uni }} \cdot \overline{\text { org } / \text { onto } / \text { teaches }}$ & 6 \\
\hline$\overline{\text { http }}: / / \overline{\text { www }} \cdot \overline{\text { uni }} \overline{1.0 r g / \text { courses/Course } 148}$ & 8 \\
\hline \multicolumn{2}{|c|}{ Graph Category $=23$} \\
\hline Binding & Number of Elements \\
\hline$? \mathrm{~L}=$ http $: / /$ www $\cdot \underline{\text { uni }} 1$. org $/$ dep $1 /$ Professor 137 & 9 \\
\hline$? \mathrm{C}=\overline{\mathrm{http}}: / / \overline{\mathrm{www}} \cdot \overline{\mathrm{uni}} \overline{1} \cdot \overline{\mathrm{org}} / \overline{\mathrm{cou}} \overline{\mathrm{cos}} / \mathrm{Course} 148$ & 8 \\
\hline \multicolumn{2}{|c|}{ Binding Category $=17$} \\
\hline
\end{tabular}

Both categories are determined by the number of elements of the object's (i.e., graph or binding) textual representation. Elements (underlined text blocks) are character sequences that are separated by either a delimiter character (e.g., ":", "/", “.”, “\#”, etc.) or by a digit following a letter character (e.g., "Course-1", "Professor137 "). Accordingly, the graph of the above example is assigned to the graph category $23(9+6+8)$ and the binding is assigned to the binding category $17(9+8)$.

Whenever a similar binding is found, it is checked whether the graphs that were published before are similar as well. Similarity is defined by means of a relation between two bindings or graphs. In our heuristic, we simply define similarity via an object's category: If and only if two objects belong to the same category, they are considered to be similar. While more complex similarity relations would obviously allow for a more accurate detection of similar objects, simple relations can usually be evaluated more efficiently.

The following two tables show a second graph that is published after the first one and the new binding that results from this publication.

\footnotetext{
${ }^{2}$ We added white space characters in the URIs for a better illustration of single elements.
} 


\begin{tabular}{|c|c|}
\hline Published Graph & Number of Elements \\
\hline $\begin{array}{l}\frac{\text { http }}{}^{1}: / / \text { www }^{1} \cdot \text { uni }^{1} \underline{1}^{1} \cdot \text { org }^{1} / \underline{\text { dep }}^{1} \underline{1}^{1} / \text { FullProfessor }^{1} \\
\text { http }^{1}: / / \text { www }^{1} \cdot \text { uni }^{1} \cdot \text { org }^{1} / \text { onto }^{1} / \text { teaches }^{1} \\
\text { http }^{1}: / / \text { www }^{1} \cdot \text { uni }^{1} \underline{1}^{1} \cdot \text { org }^{1} / \text { courses }^{1} / \text { Course }^{1}\end{array}$ & $\begin{array}{l}9 \\
6 \\
8\end{array}$ \\
\hline & ategory $=23$ \\
\hline Binding & Number of Elements \\
\hline 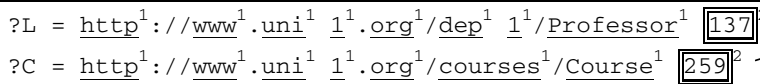 & $\begin{array}{l}9 \\
8\end{array}$ \\
\hline
\end{tabular}

According to our heuristic, both bindings as well as both published graphs are similar. The binding's and graph's elements are now further separated into two groups:

- Structure elements: These elements (almost) always remain the same for all members of a category. They represent a common structure of members of a category.

- Key elements: These elements exhibit high diversity. They represent the distinguishing parts of members of a category.

In order to determine the group of an element, a history of added elements is kept for each category. If the n-th element of a graph or binding has not yet been added at the same position before, then the diversity index of this position is increased by one (shown as superscript number). As long as the diversity index of an element does not exceed a certain threshold (here $<2$ ), it is considered to be a structure element. As soon as it exceeds the threshold, it is considered to be a key element (shown as framed text block).

If all key elements of a binding can also be found in the published graph, then a new mapping entry is generated. The following mapping entry defines that the publication of a graph of graph category 23 has caused the detection of a new binding of binding category 17 for the query of channel "NewCourseChannel". The key elements of the binding at positions 9 and 17 were found in the published graph at the positions 9 and 23, respectively.

\begin{tabular}{|l|l|l|l|}
\hline Graph Category & Channel (Query) & Binding Category & Key Element Mappings \\
\hline$\ldots$ & $\ldots$ & $\ldots$ & $\ldots$ \\
\hline 23 & NewCourseChannel & 17 & $\mathrm{BC}(9)=\mathrm{GC}(9), \mathrm{BC}(17)=\mathrm{GC}(23)$ \\
\hline
\end{tabular}

When the next graph is published, it is looked up in the mapping table whether it already contains entries for similar graphs, i.e. for the respective graph category. If this is the case, candidate bindings are generated by taking the structure elements of the binding category and the key elements of the published graph.

The described heuristic will, obviously, not always find all new bindings. The hit ratio highly depends on particular characteristics of the registered channel's query. The heuristic will probably find most new bindings for queries with high structural similarities between published graphs and found new bindings (independently of the complexity of the required querying and reasoning task), but it may not be able to find any new bindings for queries with indirect relations between the key elements of the 
published graph and the new bindings. For example, it will be difficult to predict new bindings of a query that reports the name of the lecturer who held the same course in the previous semester, since the newly published graph does not contain any information about this lecturer. In the worst case, a certain (configurable) amount of time is spent for testing and analysis without finding any new bindings. However, we saw that in many applications that build upon semantic technologies, a large number of queries exhibit high similarity between published graphs and new bindings and our approach can therefore help to significantly improve the performance of these applications.

\section{Evaluation}

In this section, we present an experimental evaluation of the proposed heuristic result set update algorithm. We therefore implemented an application scenario based on the LUBM benchmark [24]. We employed SENS to manage organizational and reference data of universities and realized client-to-client interaction by publishing knowledge and subscribing to knowledge updates. For populating the knowledge base, we took the data set of $\operatorname{LUBM}(10)\left(\sim 10^{6}\right.$ explicit statements $)$ and split it into $n=114789$ separate graphs, each of which containing 2-20 semantically related statements. Graphs describing resources of the same type differ in size, structure and order of statements. This allows us to investigate whether the heuristic is capable to handle a certain level of "noise" in the published data. In the test scenario, we published $n-$ 1000 graphs to SENS in random order. We then created a semantic event channel for each of the 14 LUBM benchmark queries and registered one subscriber at each channel. Finally, we published the remaining 1000 graphs and measured the average notification time of the resulting notifications and the publication throughput of the system.

In the test setting, SENS was run with SwiftOWLIM 2.9 [25], a semantic repository with a native full materialization ${ }^{3}$ rule entailment engine that supports RDFS [26], OWL DPL [27], OWL Horst and most features of OWL Lite (cf. [25]). All tests were run on a Pentium IV HT 3,2GHz, 4GB RAM Windows Vista server.

\subsection{Notification Time}

For our measurements, we implemented a benchmark framework that executes all tests in two runs. In the first run, SENS is started without heuristic result set updates and with a query execution phase for each publication operation. The framework records which publication operation preceded a notification, assigns each operation a unique ID and stores benchmark configuration and reference data to an XML file. In the second run, the framework restarts the Java virtual machine, loads the configuration and runs the benchmark using the heuristic result set update algorithm. By comparing the results with the results of the first run, notifications can be traced back to the publication operations they resulted from, even if multiple graphs are published at once.

The notification time benchmark results are shown in Table 1.

\footnotetext{
${ }^{3}$ Materialization can be switched off for transitive closure to reduce memory consumption.
} 
Table 1. LUBM(10)/SwiftOWLIM 2.9 Notification Times

\begin{tabular}{cccccccccc}
\hline \hline & Num. of & \multicolumn{2}{c}{ Tests } & \multicolumn{2}{c}{ Pos. Hitrate } & Mapping & \multicolumn{2}{c}{ Avg. Notification Time } \\
Channel & Notif's & Positives & Negatives & (Pos./Notifs $)$ & Heur. vs. Std. & $\begin{array}{c}\text { Heuristic } \\
\text { Entries }\end{array}$ & Standard & Heuristic & $\begin{array}{c}\text { (-1+Heur./Std.) } \\
\text { Overhead }\end{array}$ \\
\hline Q1 & 1 & 0 & 0 & $0 \%$ & 0 & $11 \mathrm{~ms}$ & $12 \mathrm{~ms}$ & $9 \%$ & $2 \mathrm{~ms}$ \\
Q2 & 0 & 0 & 0 & $0 \%$ & 0 & $0 \mathrm{~ms}$ & $0 \mathrm{~ms}$ & $0 \%$ & $4 \mathrm{~ms}$ \\
Q3 & 1 & 0 & 0 & $0 \%$ & 0 & $10 \mathrm{~ms}$ & $10 \mathrm{~ms}$ & $0 \%$ & $3 \mathrm{~ms}$ \\
Q4 & 2 & 0 & 0 & $0 \%$ & 0 & $12 \mathrm{~ms}$ & $12 \mathrm{~ms}$ & $0 \%$ & $1 \mathrm{~ms}$ \\
Q5 & 74 & 51 & 13 & $69 \%$ & 9 & $18 \mathrm{~ms}$ & $14 \mathrm{~ms}$ & $-22 \%$ & $1 \mathrm{~ms}$ \\
Q6 & 454 & 423 & 0 & $93 \%$ & 13 & $526 \mathrm{~ms}$ & $57 \mathrm{~ms}$ & $-89 \%$ & $64 \mathrm{~ms}$ \\
Q7 & 11 & 2 & 123 & $18 \%$ & 2 & $12 \mathrm{~ms}$ & $12 \mathrm{~ms}$ & $0 \%$ & $1 \mathrm{~ms}$ \\
Q8 & 487 & 458 & 0 & $94 \%$ & 12 & $148 \mathrm{~ms}$ & $19 \mathrm{~ms}$ & $-87 \%$ & $1 \mathrm{~ms}$ \\
Q9 & 14 & 0 & 48 & $0 \%$ & 3 & $381 \mathrm{~ms}$ & $394 \mathrm{~ms}$ & $3 \%$ & $23 \mathrm{~ms}$ \\
Q10 & 1 & 0 & 0 & $0 \%$ & 0 & $11 \mathrm{~ms}$ & $12 \mathrm{~ms}$ & $9 \%$ & $1 \mathrm{~ms}$ \\
Q11 & 18 & 16 & 0 & $89 \%$ & 1 & $19 \mathrm{~ms}$ & $12 \mathrm{~ms}$ & $-37 \%$ & $1 \mathrm{~ms}$ \\
Q12 & 1 & 0 & 0 & $0 \%$ & 0 & $13 \mathrm{~ms}$ & $14 \mathrm{~ms}$ & $8 \%$ & $1 \mathrm{~ms}$ \\
Q13 & 0 & 0 & 0 & $0 \%$ & 0 & $0 \mathrm{~ms}$ & $0 \mathrm{~ms}$ & $0 \%$ & $1 \mathrm{~ms}$ \\
Q14 & 359 & 344 & 0 & $96 \%$ & 7 & $447 \mathrm{~ms}$ & $31 \mathrm{~ms}$ & $-93 \%$ & $13 \mathrm{~ms}$ \\
\hline Q1-14 & 1423 & 1294 & 184 & $91 \%$ & 47 & $813 \mathrm{~ms}$ & $95 \mathrm{~ms}$ & $-88 \%$ & $21 \mathrm{~ms}$ \\
\hline \hline
\end{tabular}

The channels with the highest average notification time in the SENS standard processing mode are Q6, Q8, Q9, and Q14 (marked rows). In this configuration, the average notification time directly corresponds to the query execution time. Hence, a notification time of $\sim 500 \mathrm{~ms}$ not only causes recognizable delays for the client but also imposes a high load on the server.

The update heuristic works best for those channels with a high number of notifications (Q5, Q6, Q8, and Q14). Since more statistical information about published graphs and found bindings can be collected, also the hit rate is improved. The resulting average notification times are reduced by $22 \%, 89 \%, 87 \%$, and $93 \%$ of the original notification times.

The tests showed that the notification times for three of the four channels with particularly long notification times in the standard mode could be significantly reduced in the heuristics mode (Q6, Q8, and Q14). The reason for this lies in the high number of notifications of these channels. On the one hand, a high number of notifications provides for better hit rates during the test phase. On the other hand, a

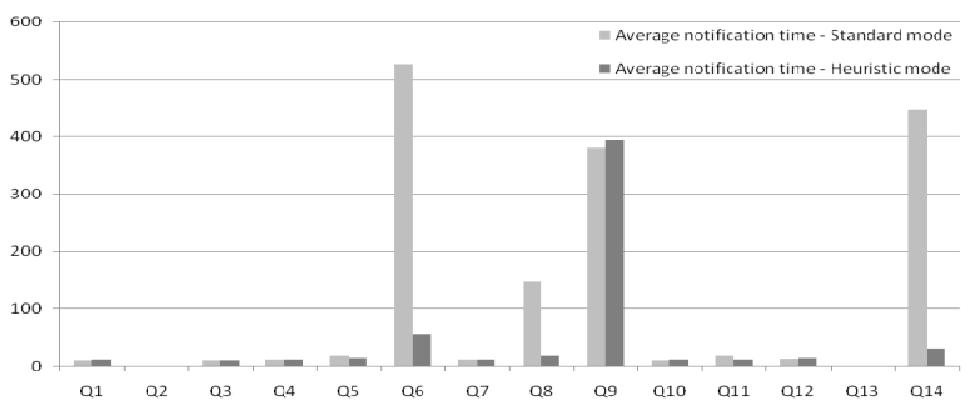

Fig. 3. Average notification times (ms) 
high number of notifications is also often the cause for high response times. The result sets of queries with many result updates grow steadily $(74,454,487$, and 359 notifications) and even if the size of a result set is decreased by deleting knowledge from the knowledge base, the average amount of contained bindings is typically high. Since the execution time of a query primarily depends on the complexity of the query and on the size of the result set, the execution time of these queries is usually higher than the execution time of those with a low number of notifications.

A comparison of response times of all 14 channels is provided in Figure 3.

Employing the result set update algorithm led to response times of less than $60 \mathrm{~ms}$ for all channels except of Q9. For Q9, the high response time of originally $381 \mathrm{~ms}$ is primarily caused by the structure and complexity of the registered query but not by a large result set (only 14 notifications). The query is characterized by the most classes and properties of all 14 LUBM queries and includes a triangular relationship between its variables. Moreover, new query results frequently contain key elements which are not contained in the published graph and this prevents the result set update heuristic from predicting correct new bindings. Consequently, the high response time in the heuristics mode results from the original processing time plus a small overhead generated by the test and analysis phases.

While the tests showed promising results with respect to the achieved notification times, it also needs to be taken into account that the test and analysis phases of the publication operations cause a certain processing overhead. Especially for those channels with a high number of mapping entries, these two phases could even last longer than the query execution phase. If this is the case, the heuristic update mechanism should either be deactivated (for the particular channel) or the number of mapping entries should be reduced, e.g., by dropping those with comparably low hit rates.

In summary, the tests showed that the most significant improvements are obtained for channels with a high number of notifications. However, the effectiveness of the heuristic also highly depends on particular characteristics of the published graphs and the registered query. Generally, the highest hit rates will be achieved when (i) key elements of bindings are typically contained in the published graphs and (ii) there is high similarity between the published graphs and the new bindings.

\subsection{Throughput}

In the previous section we showed that the proposed query result update heuristic reduces response times of certain kinds of queries. However, employing the update heuristic does not yet improve the throughput of the system. For this purpose, the collected statistics can be leveraged in another way: If most of the new bindings are found by the update heuristic, then the query processing phase could be skipped in certain intervals. In many cases, query processing is the most costly task of publication processing and skipping this phase would save the according processing time.

Figure 4 and Figure 5 show how many of the previous 10 notifications were found by the update heuristic for the channels Q5 and Q6, respectively. Both figures show the last 1000 publication operations. 


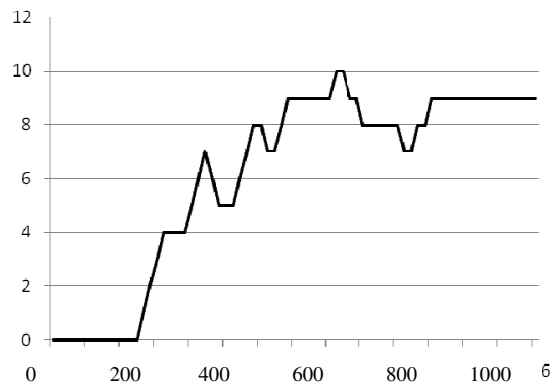

Fig. 4. Hits per 10 Notifications (Q5)

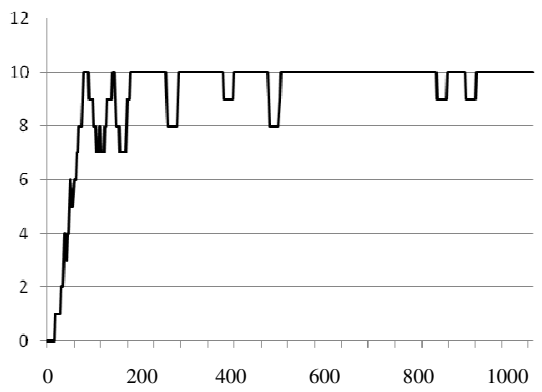

Fig. 5. Hits per 10 Notifications (Q6)

For channel Q5, the number of found bindings increases until the $400^{\text {th }}$ publication operation and then remains between 7 and 10. If the query processing would only be executed in certain greater intervals, an average of $\sim 20 \%$ of the notifications would not be found immediately. These notifications would be sent to the subscribers after the next query processing phase and exhibit essentially increased notification times. In contrast, the number of found bindings for channel Q6 increases until publication number 100 and after about 200 publication operations, the update heuristic finds almost all new bindings. Consequently, skipping the query processing for this channel introduces only a low risk of missing new bindings.

We implemented a simple extension for SENS that records the rate of notifications per publication as well as the rate of notifications found by the update heuristic. As an experimental configuration, we defined that if a channel exhibits an average of less than 0.1 notifications per publication or the update heuristic finds more than $80 \%$ of the new bindings, then the query processing is skipped for up to 10 publication processing cycles. Table 2 shows a comparison of average notification time, average time required for processing a publication, and the publication throughput (processed publication operations per second) for all three configurations: SENS standard mode, SENS with heuristic result set updates, and SENS with heuristic result set updates and applying the throughput optimization configuration.

Table 2. LUBM(10)/SwiftOWLIM 2.9 Throughput

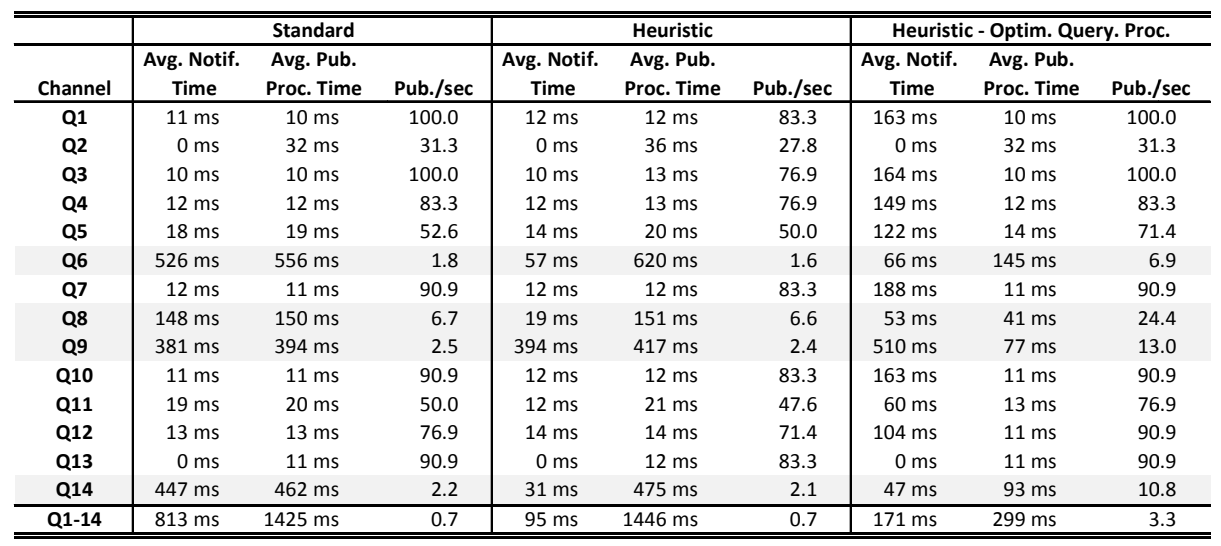


The channels with the highest average publication processing times in both SENS standard and heuristics mode are Q6, Q8, Q9, and Q14 (marked rows).

As expected, the throughput optimization extension works best for channels with a hit rate greater than $80 \%$ (see Table 1), namely Q6, Q8, and Q14. For these three channels, the publication rate increased by a factor of 3.7-5.0. Since the extension also skips query processing cycles for channels with a low notification frequency, the publication throughput of Q9 could also be increased from 2.5 to 13.0 publications per second (factor 5.1).

For the other channels, only small throughput improvements could be achieved. As query processing takes only a view milliseconds for these channels, the overhead introduced by the testing and analysis phase is almost as high as the time saved by skipping a query processing cycle. Furthermore, these channels exhibit essentially worse notification times, as SENS requires up to 10 processing cycles until it finds a new binding.

In summary, we saw that there is a trade-off between notification time and system throughput for all 14 channels. By skipping query processing cycles, more operations can be processed, but the probability of missing notifications increases accordingly. Concluding from the implemented tests, skipping query processing cycles is thus best applied for channels with (i) high query processing times and (ii) either low notification frequency or a high percentage of notifications found by the update heuristic.

\section{Conclusion}

In this paper, we introduced a heuristics framework for incremental result set updates for continuous queries on highly dynamic knowledge bases. We presented a simple heuristic for this framework and described how the collected statistical information can be employed to improve responsiveness and throughput of the semantic subscription system. We conducted an experimental evaluation based on a concrete application scenario, which demonstrated that the approach can successfully be employed to reduce response times of continuous queries by up to $90 \%$ of the original response times. Moreover, by leveraging the collected statistics for optimizing the query execution intervals, throughput could be improved by a factor of 3.7-5.1 for the most heavyweight publication operations.

Nonetheless, we also saw that the performance improvement highly depends on the concrete query. Consequently, we plan to further investigate the behavior of the heuristic in other benchmark environments as well as in real world applications in order to derive more detailed characteristics of applications for which the heuristic approach is best applied. Moreover, future work will include the improvement of the heuristic algorithm and the development of a framework extension that automatically selects the most suitable out of a collection of heuristics based on previously collected statistics.

Acknowledgements. We would like to thank Vesna Sesum-Cavic and Laszlo Keszthelyi for their valuable suggestions and numerous comments on this work. 


\section{References}

1. Nixon, L.J.B., Teymourian, K., Krummenacher, R., Moritsch, H., Momtchev, V., Ghioni, A., Schütz, A.: Semantic Clustering and Self-Organisation in Triple Space, TripCom Research Deliverable (2008), http: / / www . tripcom. org / docs / del / D2 . 4 .pdf

2. Catarci, T., Lenzerini, M.: Representing and Using Interschema Knowledge in Cooperative Information Systems. Journal of Intelligent and Cooperative Information Systems 2(4), 375-398 (1993)

3. Murth, M., Kühn, e.: A Semantic Event Notification Service for Knowledge-Driven Coordination. In: Proc. of 1st Int'l. workshop on emergent semantics and cooperation in open systems (ESTEEM), cooperation with the 2nd Int'l. Conf. on Distributed EventBased Systems (DEBS 2008), Rome, Italy, July 1 (2008)

4. Petrovic, M., Liu, H., Jacobsen, H.: G-ToPSS: Fast Filtering of Graph-based Metadata. In: Proceedings of the 14th International Conference on World Wide Web (WWW 2005). ACM Press, New York (2005)

5. Wang, J., Jin, B., Li, J.: An ontology-based publish/Subscribe system. In: Jacobsen, H.-A. (ed.) Middleware 2004. LNCS, vol. 3231, pp. 232-253. Springer, Heidelberg (2004)

6. Murth, M., Kühn, e.: Knowledge-based Coordination with a Reliable Semantic Subscription Mechanism. In: Proc. of 24th ACM Symposium of Applied Computing (SAC 2009) - Special Track on Coordination Models, Languages and Applications. ACM Press, Honolulu (2009)

7. Nixon, L.J.B., Simperl, E., Krummenacher, R., Martin-Recuerda, F.: Tuplespace-based Computing for the Semantic Web: A Survey of the State-of-the-Art. The Knowledge Engineering Review 23(2) (2008)

8. Halaschek-Wiener, C., Parsia, B., Sirin, E.: Description logic reasoning with syntactic updates. In: Meersman, R., Tari, Z. (eds.) OTM 2006. LNCS, vol. 4275, pp. 722-737. Springer, Heidelberg (2006)

9. Baader, F., Calvanese, D., McGuinness, D.L., Nardi, D., Patel-Schneider, P.F.: The Description Logic Handbook: Theory, Implementation, Applications. Cambridge University Press, Cambridge (2003)

10. Khushraj, D., Lassila, O., Finin, T.W.: sTuples: Semantic Tuple Spaces. In: 1st Ann. Int'1. Conf. on Mobile and Ubiquitous Systems (2004)

11. Fensel, D., Krummenacher, R., Shafiq, O., Kühn, e., Riemer, J., Ding, Y., Draxler, B.: TSC - Triple Space Computing, In Special issue on ICT research in Austria. Journal of Electronics \& Information Technology (e\&i Elektrotechnik \& Informationstechnik) (January-February 2007)

12. Nixon, L.J.B., Paslaru Bontas Simperl, E., Antonenko, O., Tolksdorf, R.: Towards Semantic Tuplespace Computing: The Semantic Web Spaces System. In: 22nd Ann. ACM Symposium on Applied Computing (2007)

13. Simperl, E., Krummenacher, R., Nixon, L.: A Coordination Model for Triplespace Computing. In: Murphy, A.L., Vitek, J. (eds.) COORDINATION 2007. LNCS, vol. 4467, pp. 1-18. Springer, Heidelberg (2007)

14. Gelernter, D.: Generative Communication in Linda. ACM Transactions in Programming Languages and Systems (TOPLAS) 7(1), 80-112 (1985)

15. Cerzza, D., Della Valle, E., Foxvog, D., Krummenacher, R., Murth, M.: Towards European Patient Summaries based on Triple Space Computing. In: Proc. of 1st European Conf. on eHealth, Fribourg, Switzerland, October 12-13 (2006)

16. Jena. Jena - A Semantic Web Framework for Java, http: // jena. sourceforge. net/ (last accessed: September 2007) 
17. Broekstra, J., Kampman, A., van Harmelen, F.: Sesame: A Generic Architecture for Storing and Querying RDF and RDF Schema. In: Horrocks, I., Hendler, J. (eds.) ISWC 2002. LNCS, vol. 2342, pp. 54-68. Springer, Heidelberg (2002)

18. ARQ - A SPARQL Processor for Jena, http: / / jena. sourceforge. net/ARQ/

19. Kaon2. Ontology management for the semantic web, http: / / kaon2. semanticweb.org/

20. Cai, M., Frank, M.: RDFPeers: A Scalable Distributed RDF Repository Based on a Structured Peer-to-Peer Network. In: 13th Int'l. Conference on World Wide Web (2004)

21. Liarou, E., Idreos, S., Koubarakis, M.: Continuous RDF Query Processing over DHTs. In: Aberer, K., Choi, K.-S., Noy, N., Allemang, D., Lee, K.-I., Nixon, L., Golbeck, J., Mika, P., Maynard, D., Mizoguchi, R., Schreiber, G., Cudré-Mauroux, P. (eds.) ASWC 2007 and ISWC 2007. LNCS, vol. 4825, pp. 324-339. Springer, Heidelberg (2007)

22. Prud'hommeaux, E., Seaborne, A.: SPARQL Query Language for RDF. W3C Recommendation (2008), http: / / www .w3 .org/TR/rdf-sparql-query/

23. Luckham, D.: The Power of Events: An Introduction to Complex Event Processing in Distributed Enterprise Systems. Addison-Wesley, Reading (2002)

24. Guo, Y., Pan, Z., Heflin, J.: LUBM: A Benchmark for OWL Knowledge Base Systems. Journal of Web Semantics 3(2), 158-182 (2005)

25. Ontotext. OWLIM Fact Sheet, http: / / www . ontotext.com/owlim/owLIMFact Sheet.pdf

26. Manola, F., Miller, E.: RDF Primer W3C-Recommend (2004), http://www.w3 .org/TR/rdf-primer/

27. McGuinness, D.L., van Harmelen, F.: OWL Web Ontology Language, W3C Recommendation (2004), http:/ /www.w3 .org/TR/owl-features / 